\title{
Audit of Renal Drug Dosing: Comparison of 2 Methods and Evaluation of Pharmacists' Knowledge, Attitudes, and Behaviour
}

\author{
Kimberley L Caouette, Colette B Raymond, Lindsay D Meyer, and Nicholas Honcharik
}

\begin{abstract}
Background: The renal dosing directive of the Winnipeg Regional Health Authority Pharmacy Program outlines an auditable pharmacy service whereby pharmacists are required to perform documentation (i.e., document their rationale) only if they do not adjust the dose of any medications listed in the directive.
\end{abstract}

Objective: To compare the suitability of manual orders (hard copy) and reports from the pharmacy information system (computer-generated) for determining pharmacists' compliance with the renal dosing directive; to measure compliance with the renal dosing directive; and to determine pharmacists' opinions about audit programs.

Methods: A retrospective audit was used to compare 400 manual orders with the corresponding orders in reports from the pharmacy information system, to determine compliance with the renal dosing directive. An e-mail survey was performed to gather pharmacists' opinions about audit programs.

Results: Of the 400 orders evaluated, 86 (22\%) required consideration of a dose adjustment. Of these, 78 (91\%) showed that dosing followed the guidelines for renal dysfunction in standard pharmacy references. Six (7\%) of 86 manual orders and $8(9 \%)$ of 86 pharmacy information system orders were not compliant with the renal dosing directive (i.e., no dosage adjustment and no documentation of rationale). Of 77 pharmacists approached, 34 (44\%) completed the survey. Most respondents (31/34 [91\%]) agreed that auditing is beneficial to patients, and the same number $(31 / 34$ [91\%]) agreed that auditing provides important information to the pharmacy program. Only 17 (50\%) were aware of medications listed in the renal dosing directive, and 14 (41\%) felt that they had received sufficient education about pharmacy directives. Most respondents (29/34 [85\%]) agreed that audits would reveal areas for improvement, and all (34/34 [100\%]) would comply with any changes required to facilitate performance of an audit if such changes did not increase workload.

Conclusions: Similar results were obtained with the 2 auditing methods used for this study (manual orders and reports from the pharmacy information system). However, pharmacists' current use of electronic documentation limits the feasibility of pharmacy information system audits. Survey respondents claimed that they were not familiar with the renal dosing directive, but they did agree that auditing clinical services is beneficial.

\section{RÉSUMÉ}

Contexte : La directive sur l'ajustement posologique chez les insuffisants rénaux du Programme de pharmacie de la Régie régionale de la santé de Winnipeg décrit un service de pharmacie vérifiable selon lequel les pharmaciens sont tenus de consigner les motifs seulement s'ils n'ajustent pas la posologie des médicaments énumérés dans la directive.

Objectif : Comparer la pertinence des ordonnances manuelles (version papier) et des rapports générés par le système informatique de la pharmacie (version électronique) pour déterminer la conformité à la directive sur l'ajustement posologique chez les insuffisants rénaux de la part des pharmaciens; mesurer le degré de conformité à cette directive; et obtenir l'opinion des pharmaciens quant aux programmes de vérification.

Méthodes : On a mené une vérification rétrospective pour comparer 400 ordonnances manuelles aux ordonnances correspondantes dans les rapports du système informatique de la pharmacie, afin de déterminer la conformité à la directive sur l'ajustement posologique chez les insuffisants rénaux. Un sondage par courriel a été envoyé aux pharmaciens pour obtenir leur opinion sur des programmes de vérification.

Résultats : Des 400 ordonnances évaluées, 86 (22\%) requéraient la prise en considération d'un ajustement posologique. De celles-ci, 78 (91\%) se sont avérées conformes aux lignes directrices sur l'ajustement posologique en cas d'insuffisance rénale dans les ouvrages de référence standards de pharmacie. Six $(7 \%)$ des 86 ordonnances manuelles et 8 (9\%) des 86 ordonnances saisies par le système informatique de la pharmacie n'étaient pas conformes à la directive (c.-à-d. aucun ajustement posologique et aucune consignation des motifs). Des 77 pharmaciens qui ont reçu le sondage, 34 (44\%) y ont répondu. La plupart des répondants (31/34 [91 \%]) étaient d'accord pour dire que la vérification est avantageuse pour les patients et la même proportion (31/34 [91\%]) l'était aussi pour dire que la vérification procure des renseignements utiles pour le programme de pharmacie. Seulement 17 (50\%) pharmaciens étaient au courant de la liste des médicaments de la directive et 14 (41\%) estimaient qu’ils avaient reçu une formation suffisante sur les directives pharmaceutiques. La plupart des répondants (29/34 [85\%]) ont affirmé que les vérifications permettraient de cerner des points à améliorer et tous (34/34 [100\%]) ont dit quils se conformeraient à tout changement nécessaire à la tenue d'une vérification, pourvu que cela n'entraîne pas d'augmentation de la charge de travail.

Conclusions : Des résultats similaires ont été obtenus à partir des deux méthodes de vérification utilisées dans cette étude (ordonnances manuelles 
Key words: renal dose adjustments, pharmacists' compliance, pharmacists' behaviours and attitudes

Can J Hosp Pharm 2012;65(4):277-284 et rapports du système informatique de la pharmacie). En revanche, l'utilisation actuelle de la consignation électronique par les pharmaciens limite la faisabilité des vérifications des systèmes informatiques de pharmacie. Les répondants au sondage ont affirmé ne pas être familiers avec la directive sur l'ajustement posologique chez les insuffisants rénaux, mais que la vérification des services cliniques est avantageuse.

Mots clés : ajustements posologiques chez les insuffisants rénaux, conformité de la part des pharmaciens, comportements et attitudes des pharmaciens

[Traduction par l'éditeur]

\section{INTRODUCTION}

$\mathrm{H}^{\circ}$ ospitals, clinics, and regional health authorities, along with the Institute for Safe Medication Practices Canada and Health Canada, are challenged to develop quality improvement methods that efficiently and accurately evaluate quality of patient care. Accreditation Canada outlines patient safety goals and organizational practices for health care organizations to follow to monitor quality and achieve positive outcomes. ${ }^{1,2}$ Monitoring quality of care via clinical auditing ensures transparency for health care professionals and patients and indicates areas to target for future quality improvement initiatives. ${ }^{3,4}$ Once the need for an audit has been established, active involvement of health care professionals, such as pharmacists, in the audit process can lead to a greater effect on professional practice, ensure that the audit process is efficient, and prevent adversarial relationships between auditors and those being audited. ${ }^{5-7}$ In addition, there is a need for involvement of other stakeholders, including organizations (i.e., hospitals and regional health authorities), to facilitate the audit process.

A successful audit accurately measures and evaluates the key features of a clinical practice. ${ }^{8}$ Quality of care is not directly measured during the audit; therefore, auditors rely on process, structure, or outcome indicators for information that can be used to monitor and evaluate the performance of the health system. ${ }^{5}$ Documentation on medication orders is a measureable process indicator that is used to monitor clinical care and compliance with organizational guidelines. Pharmacists working in the Winnipeg Regional Health Authority (WRHA) are required to perform documentation on the patient profile in the pharmacy information system or on hard-copy medication orders if an intervention, such as a dose adjustment, is necessary at the time of order entry, except in certain specified situations where documentation by exception applies.

The WRHA Pharmacy Program has an auditable renal dosing directive for medications that may cause harm for patients with renal dysfunction. Appropriate dosing of medications for these patients is worthy of evaluation because inappropriate dosing could result in toxic effects. ${ }^{9}$ Although conducting an audit can provide insight into opportunities to improve the quality of pharmacy care, pharmacists may not agree to participate. ${ }^{10-12}$ Limited research to date suggests that pharmacists lack enthusiasm for clinical auditing, see auditing as threatening or as an extra job, or believe that auditing is a process that may lead to finger-pointing. ${ }^{10-12}$ Since clinical audits can be costly and time-consuming, it is desirable to identify an audit method that is cost-effective, efficient, and accurate while also being acceptable to pharmacists. The purpose of this study was 3-fold: to compare the suitability of manual orders (hard copy) and reports from the pharmacy information system (computer-generated) for determining compliance with the institution's renal dosing directive; to measure compliance with the directive; and to determine pharmacists' opinions about audit programs.

\section{METHODS}

A retrospective audit was conducted to compare manual orders with the corresponding orders in the pharmacy information system report and to determine compliance with the renal dosing directive of the WRHA Pharmacy Program. The convenience sample size was based on the calculation used for binary outcome equivalence trials. With a specified significance level of 0.05 , a power of $80 \%$, an estimated proportion of $50 \%$, and a margin of equivalence of $10 \%$, the estimated sample size to determine equivalence was 395 orders in each audit. The estimated proportion of $50 \%$ was based on the estimated number of pharmacists complying with the documentation requirements outlined in the renal dosing directive. In addition to the audit, a survey was conducted to evaluate knowledge, attitudes, and behaviours of pharmacists in relation to auditing of clinical practice.

Pharmacists in the WRHA Pharmacy Program are required to assess all new medication orders to identify medications listed in the renal dosing directive (Table 1), which could cause harm to patients with compromised renal function. ${ }^{13}$ 
Table 1. Orders Requiring Consideration of Dose Adjustment in an Audit of $\mathbf{4 0 0}$ Consecutive Orders

\section{Medications Listed in Renal Dosing Directive}

Anticoagulants: dalteparin, enoxaparin, fondaparinux

Anti-infectives: acyclovir, ceftazidime, ciprofloxacin, cotrimoxazole,

fluconazole, gentamicin, levofloxacin, meropenem, nitrofurantoin,

piperacillin, piperacillin/tazobactam, tobramycin, vancomycin

Other: allopurinol, colchicine, digoxin, metformin, probenecid

Total

*Because of decreased renal function.

More specifically, the dose of and schedule for these selected medications are assessed for all patients 65 years of age or older and for patients with known diagnosis of renal dysfunction or renal failure. For patients older than 16 years, renal function is assessed by an estimation (Cockcroft-Gault non-weight-based equation normalized to $72 \mathrm{~kg}$ ) based on a serum creatinine result obtained within the 7 days before the date of the order or actual (measured) creatinine clearance. Dosing guidelines for medications administered to patients with renal dysfunction are available in the WRHA Clinical Handbook of Pharmacotherapy, ${ }^{14}$ the Micromedex database, ${ }^{15}$ and other standard pharmacy references. The outcome of the initial dosing assessment and any intervention are to be documented on manual orders or within the pharmacy information system. Documentation is by exception (i.e., required only when a dose adjustment has not been performed) and follows a standard format: drug name; estimated value of creatinine clearance; reason for not changing the dose according to the renal dosing directive, such as "prescriber refused to change dose", "prescriber did not return call/page", or "patient dehydrated-will recheck creatinine within $24 \mathrm{~h}$ ". The renal dosing directive ${ }^{13}$ is accessible to all pharmacists via the WRHA intranet website.

The study population was a convenience sample of 400 consecutive orders (beginning September 1, 2010) for all medications listed in the renal dosing directive. Orders eligible for inclusion had been processed by centralized dispensary and ward pharmacists for adult inpatients 19 years of age or older for whom a serum creatinine result was available and who had been admitted to a medical, surgical, women's, or rehabilitation unit at a tertiary care hospital within the WRHA. Orders were excluded if no serum creatinine level was available in the hospital's laboratory system within 7 days before or $24 \mathrm{~h}$ after the time of the order. Medication orders for patients in the emergency department were also excluded because many of these orders were not reviewed by a pharmacist. Orders for patients in the intensive care units were excluded because such orders were filled by a satellite pharmacy within the ICU, with a different population of pharmacists. Orders that did not relate to the first in-hospital dose of a medication and those for one-time dosing were also excluded.
For each medication order included in the audit, the name of the drug, the dose, and the dosing interval were obtained from both the manual order and the corresponding entry in the pharmacy information system report (a computer-generated report arising from medication order entry into the system). In addition, the patient's health record number, age, and sex, the form and route of administration, and the duration of therapy were accessed using the pharmacy information system. The most recent serum creatinine result (in relation to the order date) was acquired from the hospital laboratory computer system. A serum creatinine level in the hospital laboratory system obtained within $24 \mathrm{~h}$ after the order was used only if documentation by exception indicated "patient dehydratedwill recheck creatinine within $24 \mathrm{~h}$ ". If there was a discrepancy between the most recent serum creatinine level and the level documented on the manual order, the latter was used for this study. Creatinine clearance was estimated using the Cockcroft-Gault equation (normalized for a $72-\mathrm{kg}$ person) for both men and women. ${ }^{16,17}$

For this analysis, only the initial dose of medications listed in the renal dosing directive of the WRHA Pharmacy Program (Table 1) was evaluated for appropriateness. A dose was considered appropriate if it was within the range indicated in the current version of the Compendium of Pharmaceuticals and Specialties, ${ }^{18}$ the Micromedex database, ${ }^{15}$ or the Lexi-Comp database. ${ }^{19}$ If there was a range of suggested doses, the maximum dose among all resources was considered appropriate. If the renal dosing directive specified a dose adjustment, we searched for documentation by exception in the manual orders and in the corresponding orders in the computer-generated reports. Orders requiring consideration of dose adjustment were sorted into the following 3 categories: no dose adjustment, with documentation of rationale; dose adjustment, with documentation of rationale; or no dose adjustment, with no documentation of rationale (i.e., not compliant with the directive). A fourth category (dose adjustment, with no documentation of rationale) was not considered for this study but will be included in future audits.

Data were compiled in Microsoft Excel by a pharmacy practice resident (K.L.C.). The proportion of orders for which 
This single copy is for your personal, non-commercial use only.

For permission to reprint multiple copies or to order presentation-ready copies for distribution, contact CJHP at cjhpedit@cshp.ca

\section{Table 2. Comparison of Noncompliant Manual Orders and Corresponding Orders in Pharmacy Information System Reports}

\begin{tabular}{|c|c|c|c|}
\hline Drug & Dose, Route, and Frequency & 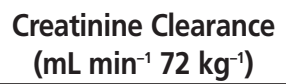 & Documentation by Exception \\
\hline Digoxin & $0.25 \mathrm{mg}$ every $24 \mathrm{~h}$ & $<10$ & No documentation \\
\hline Fluconazole & 800 mg every $24 \mathrm{~h}$ & 32 & Manual order only* \\
\hline Levofloxacin & 500 mg IV every $24 \mathrm{~h}$ & 48 & No documentation \\
\hline Meropenem & 500 mg IV every 6 h & 48 & No documentation \\
\hline Vancomycin & 1 g IV every 12 h & 56 & Manual order only* \\
\hline Vancomycin & $1 \mathrm{~g} \mathrm{IV}$ every $12 \mathrm{~h}$ & 63 & No documentation \\
\hline Vancomycin & $1 \mathrm{~g}$ IV every $12 \mathrm{~h}$ & 66 & No documentation \\
\hline Vancomycin & $1 \mathrm{~g} \mathrm{IV}$ every $12 \mathrm{~h}$ & 69 & No documentation \\
\hline
\end{tabular}

*Pharmacist recommended reducing the dose, but prescriber was unwilling to do so.

a dose adjustment was indicated but not performed was determined. Cohen's kappa coefficient was calculated to determine the magnitude of agreement between the audit of manual orders and the audit of the pharmacy information system reports.

All staff pharmacists working at the tertiary care hospital where the audit was conducted were contacted via workplace e-mail with an invitation to participate in the survey, followed by 2 e-mail reminders. Pharmacy managers and pharmacy students were excluded. The survey consisted of questions to obtain information about 3 domains related to clinical practice audits, specifically pharmacists' knowledge of, attitudes toward, and behaviours related to such audits. The questions were based upon literature about pharmacists' perceptions of audits ${ }^{11,12,20}$ and were field-tested by 6 pharmacists to evaluate face and content validity. Respondents were asked to rate their agreement with various statements on a 5-point Likert scale, where $1=$ strongly agree and $5=$ strongly disagree. The survey was open to electronic response via www.surveymonkey.com for 4 weeks in October 2010.

A confirmatory factor analysis was performed on the survey responses using SAS PROC CALIS (Covariance Analysis of Linear Structural Equations) to assess whether the questions about attitudes and behaviours measured separate constructs and whether those constructs adequately explained the variability in the sample data. Because the responses were based on a Likert scale, a diagonally weighted least-squares method was used to determine goodness of fit. Analyses were conducted with SAS software, version 9.0 (SAS Institute Inc, Cary, North Carolina).

The University of Manitoba Research Ethics Board approved this project, and consent was not required to abstract patient data. Survey participants consented to participate in that part of the study and were made aware that their participation was anonymous and voluntary.

\section{RESULTS}

A total of 400 manual orders (hard copy) and the corresponding orders in the pharmacy information system report met the inclusion criteria and were reviewed for appropriateness of dose. Of the 400 manual orders, 86 (22\%) required consideration of a dose adjustment because of decreased renal function. The patients for whom those orders had been prepared were predominantly male (51/86 [59\%]), their mean age was 57 years, and for about half of them (46/86 [53\%], the mean calculated creatinine clearance was 30 $\mathrm{mL} / \mathrm{min}$ or less.

Documentation of renal dose adjustment on the manual order was compared with documentation of adjustment in the pharmacy information system report. For 78 (91\%) of the 86 orders (both manual and computer-generated), the dose and frequency of medications listed in the renal dosing directive (Table 1) were appropriate, and no dose adjustment was required. The remaining 8 medication orders (9\%) were deemed to require an adjustment (Table 2). For 2 of the manual orders ( $2 \%$ of the total 86 orders), the reason for not adjusting the dose was documented, and the orders were judged to be compliant with the renal dosing directive. The remaining 6 manual orders $(7 \%$ of the total) and the 8 computer-generated orders ( $9 \%$ of the total) were judged to be noncompliant with the renal dosing directive, as no interventions were performed, and no documentation was found. Serum creatinine and estimated creatinine clearance were documented on all noncompliant manual orders, but this information did not explain the rationale for not adjusting the dose.

To determine the level of agreement between the 2 auditing methods, Cohen's kappa coefficient was calculated for the manual orders and the corresponding orders in the pharmacy information system report. Kappa coefficients between 0.81 and 1.00 represent "very good" strength of agreement, and the calculated coefficient in this analysis was 0.84 , within this range. Therefore, the 2 audits (based on manual orders and pharmacy information system reports) had similar performance. ${ }^{21}$

Of 77 pharmacists who were contacted to participate in the survey, 34 (44\%) completed the survey. All of the respondents held a permanent position, 29 (85\%) had worked in their current position for 2 years or longer, and 22 (65\%) had been 
pharmacists for 10 years or more. Only $17(50 \%)$ claimed to be aware of the renal dosing directive, and $11(32 \%)$ felt that they were familiar with the expectations of the directive (Table 3). Most respondents (31 [91\%]) agreed that auditing was beneficial for patients, $29[85 \%])$ agreed that auditing is a way to monitor pharmacists' compliance with directives, and 31 (91\%) agreed that auditing provides important information to the pharmacy program. Only 14 (41\%) felt that they had received sufficient education about pharmacy program directives. Few (11 [32\%]) felt that auditing pharmacists' documentation (i.e., documentation by exception) on manual orders would be sufficient to evaluate renal drug dosing services, and none felt that auditing only the computergenerated reports from the pharmacy information system would be accurate. Most respondents (29 [85\%]) agreed that audits would reveal areas for improvement, and all stated that they would comply with practice changes needed to facilitate an audit if it did not increase their workload.

Timing is critical to ensure that the need for improvements suggested by an audit is recognized and appropriate

Table 3. Results of a Survey of Pharmacists' Knowledge, Attitudes, and Behaviours Related to Clinical Audits

\section{Statement on Survey}

\section{No. (\%) of Respondents in Agreement*} $(n=34)$

\section{Knowledge}

I am aware of which medications are in the WRHA renal dosing directive.

I am familiar with the pharmacist expectations outlined in the WRHA renal dosing directive.

\section{Attitudes}

The purpose of conducting an audit is to improve patient care.

The results of an audit are educational.

An audit based on documentation found on the hard copy of the order would provide accurate results.

Auditing is beneficial to patients.

Auditing is beneficial to pharmacists.

An audit has practical value for pharmacists.

Auditing provides important information to the WRHA Pharmacy Program.

The purpose of conducting an audit is to monitor that pharmacists are meeting practice expectations.

An audit based on documentation found in the pharmacy information system would provide accurate results.

Auditing is a necessary component of providing high-quality patient care.

I have received sufficient education on the contents of the WRHA renal dosing directive to be adherent to this directive.

I have received sufficient education on the contents of all WRHA pharmacy program directives to be adherent to these directives.

The expectations of pharmacists outlined in the WRHA renal dosing directive require a reasonable amount of time.

An audit based on documentation is a sufficient method to evaluate renal drug dosing services.

Documentation by exception is the appropriate method of documentation for the WRHA renal dosing directive.

An audit of pharmacist renal drug dosing services is a nonthreatening process.

\section{Behaviours}

If I was aware that my work was being audited for adherence to the WRHA renal dosing directive, I would perform differently than I usually would.

If I was required to alter the way I documented my work in a manner that DOES NOT increase pharmacist workload in order to facilitate an audit of clinical services, I would comply.

If I was required to alter the way I documented my work, for a limited period of time, in a manner that DOES NOT increase pharmacist workload in order to facilitate an audit of clinical services, I would comply.

If an audit revealed some areas for improvement of pharmacist clinical services, I would be influenced to alter the way I practice.

\begin{tabular}{|c|c|c|}
\hline 17 & (50) & $3.2 \pm 1.3$ \\
\hline 11 & (32) & $2.8 \pm 1.1$ \\
\hline 32 & (94) & $4.2 \pm 0.5$ \\
\hline 31 & (91) & $4.2 \pm 0.5$ \\
\hline 8 & (24) & $2.9 \pm 0.8$ \\
\hline 31 & (91) & $4.2 \pm 0.5$ \\
\hline 27 & (79) & $3.9 \pm 0.5$ \\
\hline 31 & (91) & $4.1 \pm 0.5$ \\
\hline 31 & (91) & $4.0 \pm 0.4$ \\
\hline 29 & (85) & $4.0 \pm 0.6$ \\
\hline 0 & & $2.1 \pm 0.8$ \\
\hline 30 & (88) & $4.0 \pm 0.5$ \\
\hline 10 & (29) & $2.7 \pm 1.2$ \\
\hline 14 & (41) & $3.0 \pm 1.2$ \\
\hline 15 & (44) & $3.5 \pm 0.7$ \\
\hline 11 & (32) & $3.0 \pm 0.8$ \\
\hline 12 & (35) & $3.2 \pm 1.0$ \\
\hline 28 & (82) & $4.0 \pm 0.7$ \\
\hline 6 & (18) & $2.5 \pm 1.1$ \\
\hline 34 & (100) & $4.2 \pm 0.4$ \\
\hline 33 & (97) & $4.1 \pm 0.4$ \\
\hline 29 & (85) & $4.1 \pm 0.8$ \\
\hline
\end{tabular}

Mean Score \pm SD $t$

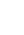


changes are adopted. Of the 34 respondents, 19 (56\%) felt that the results of an audit should be communicated to staff within 3 months of the audit being performed. Respondents had no strong opinions about who should conduct an audit.

According to the goodness-of-fit index, the 2 factors examined (attitudes and behaviours) fit the data well $\left(R^{2}=\right.$ 0.9959), which suggests that the questions about attitudes and behaviours assessed different constructs. However, the standardized root mean square residual $(\mathrm{SRMR}=0.2132)$ was greater than expected, which suggests less-than-optimal fit..22 This outcome may have resulted from the small sample size.

\section{DISCUSSION}

This retrospective study compared 2 clinical audit methods for renal dose adjustments performed by pharmacists. In the majority (over 90\%) of orders that required an adjustment because of renal dysfunction, the medication was determined to have been correctly dosed.

The second component of this study was a survey to evaluate knowledge, attitudes, and behaviours of pharmacists related to clinical practice audits. Responses to the survey indicated that knowledge of the dosing directive was poor, whereas attitudes about the benefits of auditing were generally positive, as were behaviours toward auditing.

Adjusting medication doses for renal dysfunction increases the number of appropriately dosed medications, which may decrease adverse drug effects, prevent extended hospital stays, and decrease health care costs.9, ${ }^{93-25}$ Most medications that required dose adjustment in this study were adjusted in accordance with the renal dosing directive. These findings were expected, as advising the prescribing physician when medications listed in the directive need adjustment is a routine task for WRHA pharmacists. This finding is similar to current literature about drug dosage optimization and compliance with dosing guidelines for patients with renal dysfunction. ${ }^{924,25}$

Estimations of renal clearance are routinely used to determine dose appropriateness for renally eliminated medications. Many factors influence decisions about adjusting the dose of a medication, including the method used to estimate renal function, patient-related factors, medication-related factors, and the particular reference consulted..$^{21,26,27}$ In this study, when deciding if the initial in-hospital dose was appropriate or required adjustment, we took a conservative approach. Because we lacked clinical information that might have influenced dose assessment, we evaluated the dosing ranges presented in multiple references to establish the maximum acceptable dose for each medication listed in the directive. We used a modified Cockcroft-Gault equation for creatinine clearance, normalized for a $72-\mathrm{kg}$ patient, instead of the modification of diet in renal disease (MDRD) or the chronic kidney disease epidemiology collaboration equation for dose adjustments. ${ }^{28}$ We could have used the MDRD or traditional Cockcroft-Gault formula but chose the modified Cockcroft-Gault equation to conform with regional pharmacy policy. The Cockcroft-Gault equation has not been studied for dose adjustments based on a single creatinine level for all medications or all patient populations. ${ }^{28}$ As such, this equation may over- or under-estimate glomerular filtration rate. The majority of the first in-hospital doses were dosed appropriately, according to available references, and both audit methods showed that either the physician selected an appropriate dose or a pharmacist provided an appropriate dose recommendation before the prescribing physician wrote the order. Neither auditing method captured all pharmacist interventions, as no documentation was available to explain the process for choosing doses that were deemed compliant with the renal dosing directive.

According to the renal dosing directive, the initial dosing assessment and intervention must be documented within the pharmacy information system. ${ }^{13}$ Serum creatinine and estimated creatinine clearance were documented on the manual orders, but there was no documentation stating that the prescribing physician had been contacted with this information. Pharmacists did not document serum creatinine, estimated creatinine clearance, or reasons for not adjusting doses when indicated in the pharmacy information system reports. In other words, documentation of rationale was provided in the manual orders or not at all. Furthermore, all of the survey respondents stated that they did not believe that auditing the computer-generated reports would generate accurate results. Unless pharmacists change current documentation practices, it will be difficult to use pharmacy information system reports to audit dose adjustments made by pharmacists to address renal impairment. Alternatively, the WRHA Pharmacy Program could consider modifying the renal dosing directive, with pharmacist input, to recommend a more user-friendly method of documentation.

Although similar proportions of manual and computergenerated orders were not compliant with the renal dosing directive, we cannot state definitively that the 2 audit methods yielded the same results. The small number of medications that required adjustment suggests that the study may have been underpowered to reveal a difference between the 2 audit methods. Pharmacists must be providing documentation within the pharmacy information system reports before this system can be used for clinical audit. As a result of this study, the WRHA is now reminding pharmacists about the renal dosing policy, and managers are working across the region's hospitals to standardize and encourage consistent documentation within the pharmacy information system.

In the survey about pharmacists' knowledge, attitudes, and behaviours related to clinical audits, a large proportion of respondents reported poor knowledge about the renal dosing directive and the requirements for compliance. All pharmacists 
have access to the directive through the institution's intranet website. In addition, every pharmacist is required to attend a mandatory, in-person, region-wide in-service session during new staff orientation and also, in most cases, whenever a directive is implemented or changed. Literature suggests that multifaceted interventions, such as a combination of manual and computer reminders, are more effective than single strategies when attempting to change practices. ${ }^{29,30}$ Electronic or computergenerated prompts or alerts are more effective than group education sessions, and peer-led or one-to-one outreach sessions also show benefit in improving medication practices. ${ }^{29,31}$ Reminder e-mail messages, computer links to resources, education sessions, and electronic alerts ${ }^{29,31}$ have been proposed as ways to improve knowledge about the renal dosing directive in the WRHA,

The results of this survey are encouraging, in that they revealed pharmacists' feelings that audit of pharmacy clinical services is beneficial to patients and to pharmacists overall. In a survey conducted in Scotland about the attitudes of community pharmacists (including staff pharmacists and managers, who accounted for $74 \%$ of all respondents) toward pharmacy practice audits, respondents agreed that an audit is beneficial (69\%) and educational (67\%) and can help patients in the long term $(63 \%) .{ }^{11}$ That survey also sought to determine the barriers that prevent pharmacists from participating in audits. The respondents agreed that factors such as lack of time, excessive workload, difficulty in finding relief workers, lack of financial provision, and lack of knowledge hindered their participation in audits. ${ }^{11}$ Overall, pharmacists who responded positively toward auditing were more likely to participate in, coordinate, and carry out audit projects. ${ }^{11,12}$ In our setting, pharmacists who completed the survey believed that auditing is nonintrusive and nonthreatening and were therefore willing to alter the way they provide documentation in order to facilitate an audit, as long as workload is not increased. For that reason, auditing other directive-based clinical services in the WRHA Pharmacy Program, such as therapeutic drug monitoring, discontinuing or preventing administration of medications contraindicated for patients with documented allergy, and medication management (in terms of effectiveness or adverse events), would likely have the support of pharmacists.

This study had several limitations. Patient details, such as stature and illness status, were not used to determine actual creatinine clearance. We were unaware of the indication for use of a particular drug or choice of dose; however, this parallels the situation of the pharmacist who conducted each original order review, who may not have had such details when assessing dose appropriateness. Some pharmacists might have chosen not to provide documentation once they had reviewed the patient's situation and determined that they agreed with the prescribed dose, since current practice is to provide documentation by exception. Adjudicating audited orders on the basis of maximal dosing without knowing the specific indication may have led to misclassification of orders as being compliant with the directive, when in fact they were not. Because few medication orders required adjustment, the study may have been underpowered to show a true difference between the audit of manual orders (hard copy) and the audit of orders as reported in the pharmacy information system. The fact that a high proportion of survey respondents (91\%) agreed that an audit would be beneficial may have been influenced by a social desirability bias; however, survey responses were anonymous, which would be expected to limit such bias. Finally, the respondents, who accounted for only $44 \%$ of those invited to participate, might not have been representative of the population, which could be considered a source of selection bias.

\section{CONCLUSIONS}

This comparison of clinical auditing methods and an accompanying survey have provided insight into pharmacists' compliance with a renal dosing directive and their knowledge, attitudes, and behaviours related to clinical auditing. We conclude that pharmacists were compliant with the WRHA renal dosing directive. Although the 2 audit methods (manual order versus pharmacy information system reports) yielded similar results, pharmacists' current documentation practices limit the use of pharmacy information system reports for audits. Overall, pharmacists who responded to the survey lacked knowledge about the renal dosing directive, had positive attitudes toward auditing, supported altering documentation practice so long as workload was not increased, and would be influenced by audit results to alter the way they practised.

\section{References}

1. Qmentum program 2009 standards: managing medication. Ottawa (ON): Accreditation Canada; 2009.

2. CCHSA patient/client safety goals and required organizational practices (ROPS): evaluation of implementation and evidence of compliance. Ottawa (ON): Canadian Council on Health Services Accreditation; 2007.

3. de Vos M, Graafmans W, Kooistra M, Meijboom B, Van Der Voort P, Westert G. Using quality indicators to improve hospital care: a review of the literature. Int J Qual Health Care 2009;21(2):119-129.

4. Hebert P. Measuring performance is essential to patient-centred care [editorial]. CMAJ 2010;182(3):225.

5. Bowen S, Kreindler SA. Indicator madness: a cautionary reflection on the use of indicators in healthcare. Healthc Policy 2008;3(4):41-48.

6. Jamtvedt G, Young JM, Kristoffersen DT, O’Brien MA, Oxman AD. Audit and feedback: effects on professional practice and health care outcomes. Cochrane Database Syst Rev 2006;(2):CD000259.

7. Beckmerhagen IA, Berg HP, Karapetrovic SV, Willborn WO. On the effectiveness of quality management system audits. TQM Mag 2004; 16(1):14-25.

8. Ellis BW, Sensky T. A clinician's guide to setting up audit. BMJ 1991; 302(6778):704-707.

9. Long CL, Raebel MA, Price DW, Magid DJ. Compliance with dosing guidelines in patients with chronic kidney disease. Ann Pharmacother 2004;38(5):853-858 
10. Ashmore S, Johnson T. Guide to significant event audit. Pharm J 2006; 277(7412):174-175.

11. Krska J. Attitudes to audit among community pharmacists in grampian. Pharm J 1994 Jul 9;253:97-99.

12. Ashmore S, Johnson T. Enthusiastic and organised teams will produce successful clinical audits. Pharm Pract 2006;16(4):110-113.

13. Assessment of initial medication orders for appropriate dosing based on renal function. Winnipeg (MB): Winnipeg Regional Health Authority Pharmacy Program; 2007.

14. Rosenthal B, Gin A. WRHA clinical handbook of pharmacotherapy. Winnipeg (MB): Winnipeg Regional Health Authority; 2004.

15. Micromedex ${ }^{\circledR}$ healthcare series [intranet database]. Version 5.1. Greenwood Village (CO): Thomson Reuters Healthcare.

16. Helou R. Should we continue to use the Cockcroft-Gault formula? Nephron Clin Pract 2010;116(3):c172-c186.

17. Ryzner KL. Evaluation of aminoglycoside clearance using the modification of diet in renal disease equation versus the Cockcroft-Gault equation as a marker of glomerular filtration rate. Ann Pharmacother 2010; 44(6):1030-1037.

18. Repchinsky C, editor. Compendium of pharmaceuticals and specialties. Ottawa (ON): Canadian Pharmacists Association; [annual publication].

19. Lexi-Drugs [database]. Hudson $(\mathrm{OH})$ : Lexi-Comp Inc.

20. Hynam B. Development of pharmaceutical audit in hospitals. Pharm J 1995 Oct 5;255:504-506

21. Chmura Kraemer C, Periyakoil VS, Noda A. Kappa coefficients in medical research. Stat Med 2002;21(14):2109-2129.

22. Schreiber JB, Nora A, Stage FK, Barlow EA, King J. Reporting structural equation modeling and confirmatory factor analysis results: a review. J Educ Res 2007;99(6):323-338.

23. Cantú TG, Ellerbeck EF, Yun SW, Castine SD, Kornhauser DM. Drug prescribing for patients with changing renal function. Am J Hosp Pharm 1992;49(12):2944-2948

24. Hassan Y, Al-Ramahi RJ, Aziz NA, Ghazali R. Impact of a renal drug dosing service on dose adjustment in hospitalized patients with chronic kidney disease. Ann Pharmacother 2009;43(10):1598-1605.

25. Falconnier AD, Haefeli WE, Schoenenberger RA, Surber C, MartinFacklam M. Drug dosage in patients with renal failure optimized by immediate concurrent feedback. J Gen Intern Med 2001;16(6):369-375.

26. Dowling TC, Matzke GR, Murphy JE, Burckart GJ. Evaluation of renal drug dosing: prescribing information and clinical pharmacist approaches. Pharmacotherapy 2010;30(8):776-786.

27. Patel N, Scheetz MH, Drusano GL, Lodise TP. Determination of antibiotic dosage adjustments in patients with renal impairment: elements for success. J Antimicrob Chemother 2010;65(11):2285-2290.

28. Moranville MP, Jennings HR. Implications of using modification of diet in renal disease versus Cockcroft-Gault equations for renal dosing adjustments. Am J Health Syst Pharm 2009;66(2):154-161.

29. Lu CY, Ross-Degnan D, Soumerai SB, Pearson SA. Interventions designed to improve the quality and efficiency of medication use in managed care: a critical review of the literature - 2001-2007. BMC Health Serv Res 2008;8:75.
30. Shea S, DuMouchel W, Bahamonde L. A meta-analysis of 16 randomized controlled trials to evaluate computer-based clinical reminder systems for preventive care in the ambulatory setting. J Am Med Inform Assoc 1996; 3(6):399-409.

31. Pearson SA, Moxey A, Robertson J, Hains I, Williamson M, Reeve J, et al Do computerised clinical decision support systems for prescribing change practice? A systematic review of the literature (1990-2007). BMC Health Serv Res 2009;9:154.

Kimberley L Caouette, BSCPharm, ACPR, was, at the time this study was completed, a Pharmacy Resident with the Winnipeg Regional Health Authority, Winnipeg, Manitoba. She is currently a Clinical Pharmacist with The Ottawa Hospital, Civic Campus, Ottawa, Ontario, and in late summer 2012 will become the Regional Liaison Pharmacist for The Ottawa Hospital Cancer Centre.

Colette B Raymond, PharmD, MSC, ACPR, is Staff Development Practice Evaluation Pharmacist with the Winnipeg Regional Health Authority Pharmacy Program and is also affiliated with the Department of Pharmaceutical Services, Health Sciences Centre Hospital, in Winnipeg, Manitoba.

Lindsay D Meyer, BSP, ACPR, CAE, was, at the time this study was completed, a Pharmacy Resident with the Winnipeg Regional Health Authority, Winnipeg, Manitoba. She is now a Clinical Pharmacist, Pediatrics, with University of Alberta Hospital, Alberta Health Services, Edmonton, Alberta.

Nicholas Honcharik, PharmD, FCSHP, is Regional Pharmacy Manager with the Winnipeg Regional Health Authority Pharmacy Program. He works in the Department of Pharmaceutical Services at the Health Sciences Centre Hospital in Winnipeg, Manitoba.

\section{Address correspondence to:}

Kimberley L Caouette

The Ottawa Hospital, Civic Campus,

1053 Carling Avenue

Ottawa ON K1Y 4E9

e-mail: kcaouette@toh.on.ca

\section{Acknowledgements}

The contribution of the University of Manitoba Biostatistical Consulting Unit and the participation of all pharmacists surveyed are gratefully acknowledged. 